\title{
Comparative Statics of a Monopolistic Firm Facing Rate-of-Return and Command-and-Control Pollution Constraints
}

\author{
Michael R. Caputo ${ }^{\S}$ \\ Department of Economics \\ University of Central Florida \\ P.O. Box 161400 \\ Orlando, FL 32816-1400 \\ email: mcaputo@bus.ucf.edu \\ fax: (407) 823-3269 \\ phone: (407) 823-1405 \\ Dmitriy Popov \\ Department of Economics \\ University of California \\ One Shields Ave. \\ Davis, CA 95616-3815 \\ email: dapopov@ucdavis.edu
}

\begin{abstract}
The intrinsic comparative statics properties of a general rate-of-return regulated, profitmaximizing model of a monopolist facing a command-and-control pollution constraint are derived. Recent advances in the theory of comparative statics are used to derive the basic comparative statics of the model, which are contained in an observable negative semidefinite matrix and possess the form of Slutsky-like expressions. We consider several command-and-control pollution constraints that are commonly implemented in practice, and conclude that the intrinsic comparative statics properties of the model are qualitatively invariant to the type of command-andcontrol pollution constraint imposed. We compare our results with those extant, and find that several basic results from the standard A-J model no longer hold in our model.
\end{abstract}

Keywords: Averch-Johnson, Comparative statics, Command-and-control regulations

JEL Codes: D21, D42, L12, L51, Q50

$\S$ Corresponding author 


\section{Introduction}

In a seminal paper titled "Behavior of the Firm Under Regulatory Constraint," Averch and Johnson (1962) studied the problem of a rate-of-return regulated, profit-maximizing monopolist. Yet in addition to facing a rate-of-return (ROR) constraint, some such firms must also comply with environmental regulations. Proper understanding of how such firms respond to changes in exogenous policy variables is crucial for the regulating agencies, which induce changes in the said variables through the constraints. ${ }^{1}$ A comparative statics analysis of the Averch-Johnson (A-J) model broadened by the inclusion of command-and-control (CAC) pollution restrictions can provide this understanding. The goal of the paper, therefore, is to derive the intrinsic comparative statics properties of an extended A-J model that includes CAC pollution constraints that are commonly implemented in practice.

A legitimate question arises: Why a model involving a CAC pollution constraint at a time when market-based instruments appear to be at the forefront of modern environmental policy and ROR regulation is being supplanted by price-cap regulation? Our answer to this question is twofold. First, although the role of CAC constraints in environmental policy has diminished since the enactment of the US 1990 Clean Air Act Amendments, the role played by CAC restrictions is still significant. ${ }^{2}$ For example, according to Perino (2010, p. 5), up through 1999 most coal-fired power plants in the United States were subject to an emission standard on sulfur dioxide at either the federal, state, or local level. Perino (2010, p. 5) further noted that investor-owned plants were also subject to ROR regulation in many states at that time. What's more, Meran and Schwalbe (2006, p. 9) indicated that in Germany, in addition to environmental standards, there is wide-

\footnotetext{
${ }^{1}$ A related matter is how the regulatory agencies determine the allowed rate-of-return in formal regulatory hearings. See, e.g., Joskow (1972), for an empirical examination of this issue.

${ }^{2}$ A detailed discussion of the issues that could determine the future of CAC constraints in environmental policy is beyond the scope of this work. Readers interested in such matters are referred to Helfand and Berck (2003) for a survey.
} 
spread use of ROR regulation to regulate waste-water treatment facilities. Thus, there is evidence that in the recent past in the United States, and at present in Germany, that there exist firms that were, or are, subject to both ROR and CAC pollution constraints.

Second, we see our paper as a precursor to the comparative statics analysis of models involving (i) price-cap regulation and CAC pollution constraints, (ii) ROR regulation and marketbased pollution constraints, and (iii) price-cap regulation and market-based pollution constraints. As a result, our model can serve as a yardstick for such extensions, as we now explain. First note that in our extended model, we have added a CAC emissions constraint to the prototype A-J model. It thus follows that all the differences between our model and the standard A-J modelincluding, most notably, their comparative statics - can be attributed to the presence of the (binding) emissions constraint, as this is what fundamentally differs between the two. Given the results for our extended model, one can then construct a model of a monopolistic firm facing the CAC emissions constraints studied here, but instead of including a ROR constraint, the model could instead include a price-cap regulatory constraint. As before, the differences in the deduced results between these two models can be fully attributed the presence of the binding price-cap regulatory constraint, seeing as the environmental constraints are identical in the two. Proceeding in this way, one can come to a full understanding of the implications that the aforesaid economic and environmental constraints have on a firm's behavior.

Although a ROR regulated firm could face many types of pollution standards, economists have traditionally studied uniform restrictions on pollution emissions. Helfand (1991, pp. 622) analyzed the CAC pollution constraints faced by actual firms and discovered that "the correspondence between the frequency of use in the literature and frequency of use in the standards is not perfect." Moreover, Helfand (1991, pp. 622, 624-625) identified four general types of pollution-control standards that occur in practice, scilicet, an upper bound on the emissions rate, an upper bound on emissions per unit of a output, an upper bound on emissions per unit of a polluting input or abatement capital, and an upper bound on a polluting input or a lower bound on abatement capital, for a total of six specific CAC environmental constraints. Accordingly, we 
consider these six pollution constraints in deriving the intrinsic comparative statics properties of our ROR and CAC constrained model of a profit maximizing monopolistic firm.

Rose et al. (1992) were the first to study the problem of a monopolist maximizing profit subject to both ROR and CAC pollution constraints. Their results hinged upon the share of abatement capital allowed in the rate base. Referring to the regulation of electricity companies in the US, Rose et al. (1992, p. 133) observed that "What must be noted is the fact that with the exception of Tennessee (which regulates no electric generating units), pollution abatement equipment is allowed to be placed in the rate base by every state commission and the FERC." Even though the paper by Rose et al. (1992) was rather comprehensive, it did not focus on the comparative statics of the model. More recently, Meran and Schwalbe (2006) developed several simple models of a firm that accounted for ROR and CAC pollution constraints, but like Rose et al. (1992), they did not derive the comparative statics of the models.

There also exists related research that examines the interplay among different regulatory agencies. A classic example of such work is the paper by Baron (1985), in which he formulated a game between an environmental regulator in charge of setting pollution control policy and a public utility commission charged with regulating the price of a monopoly, and then examined cooperative and Stackelberg equilibria of the game. Though Baron (1985, Appendix) derived the comparative statics of the two equilibria for a tightly specified version of the game, he did not examine how the regulated monopoly behaved in the face of the two regulations. Indeed, the derivation of comparative statics in models of monopolistic firms that include multiple forms of regulation is lacking in the regulatory literature. Evidence supporting this claim can be found in the comprehensive survey by Armstrong and Sappington (2007), seeing as they mentioned the words "comparative statics" but once, in footnote 147. Furthermore, and as expanded upon below, the comparative statics we derive are observable and hence empirically testable, so they are not just theoretical curiosities but instead are properties fundamental to the model that can be statistically tested. 
Ostensibly, the main result of Averch and Johnson (1962) is the so-called A-J effect, which states that if a monopoly is subject to a ROR constraint, then the amount of the regulated input it uses increases as the allowable ROR on the regulated input decreases. But Caputo and Partovi (2002, pp. 3-7 and Lemma 2) demonstrated that the A-J effect is actually not intrinsic to the archetype A-J model, and derived four necessary and sufficient conditions for it to hold and identified other sufficient conditions that imply it. Given these results, it is therefore not surprising that the A-J effect is not intrinsic to our extended version of the A-J model either.

In related work, Caputo and Partovi (2008, Theorem 1) showed that the comparative statics expression that represents the A-J effect plays an integral role in determining the form and economic interpretation of the fundamental comparative statics of the prototype A-J model. In the extended version of the A-J model contemplated here, however, the role played by the analogous comparative statics expression is greatly diminished in determining its intrinsic comparative statics properties because of the binding CAC emissions constraint.

Several other substantive conclusions emerge from our analysis, two of which we mention here. First, the intrinsic comparative statics of our extended model are more complex in form than those derived from the standard A-J model. Nonetheless, the intuitive Slutsky-like appearance of them is retained, albeit in a generalized form. Moreover, the comparative statics of our extended model are in principle observable, i.e., measurable. This is important seeing as it implies that one can carry out a full empirical test of the extended model's intrinsic qualitative properties. As a result, the testable implications of our model can be empirically scrutinized to determine if a sample of firms is indeed behaving in a manner that is consistent with the underlying theory. This property of an economic model is of more than passing relevance to regulators if they are using the model to prognosticate the effects that regulatory policy changes have on the affected firms.

Second, the aforesaid comparative statics are qualitatively invariant to the type of CAC pollution constraint that binds. That is, the form of the negative semidefinite matrix characterizing the intrinsic comparative statics of our extended model is the same regardless of the type of 
CAC pollution constraint imposed. This refutes, in one sense, the claim by Helfand (1991, p. 622) that "Standards with such varied forms must inevitably provide firms with different incentives," seeing as the basic comparative statics of the model are qualitatively identical regardless of the type of binding CAC pollution constraint in place. These and other substantive results, as well as those of a more technical nature, that differ from those implied by the standard A-J model are derived in what follows, and an intuitive discussion of them is provided.

\section{Model Formulation and Preliminary Results}

A profit-maximizing monopolist employs $(M+1)$ inputs $\mathbf{x}_{M+1} \in \mathbb{R}_{+}^{M+1}$ in order to produce one homogeneous output $y \in \mathbb{R}_{+}$. Moreover, and without loss of generality, input $(M+1)$ is regulated. An important feature of our model is that the firm has an option of purchasing abatement capital $x_{M+2} \in \mathbb{R}_{+}$. The complete input and abatement capital vector is written as $\mathbf{x}_{M+2} \in \mathbb{R}_{+}^{M+2}$, and the corresponding vector of competitive prices is denoted by $\mathbf{w}_{M+2} \in \mathbb{R}_{++}^{M+2}$. The $C^{(2)}$ production function is denoted by $f(\cdot): \mathbb{R}_{+}^{M+1} \rightarrow \mathbb{R}_{+}$, with partial derivatives $f_{x_{l}}\left(\mathbf{x}_{M+1}\right)>0$, $l=1,2, \ldots, M+1$. The monopolist faces a $C^{(2)}$ inverse demand function $P(\cdot): \mathbb{R}_{+} \rightarrow \mathbb{R}_{+}$, thus the revenue function $R(\cdot)$ is $C^{(2)}$ and defined by $R\left(\mathbf{x}_{M+1}\right) \stackrel{\text { def }}{=} P\left(f\left(\mathbf{x}_{M+1}\right)\right) f\left(\mathbf{x}_{M+1}\right)$.

We use the essential elements of Rose et al.'s (1992) construction to set up our ROR constraint. Rose et al. (1992) placed the abatement capital in the rate base. In order to do the same in our work, we introduce two parameters. Let $s \in \mathbb{R}_{++}$denote the allowable ROR on both regulated and abatement capitals, and let $\alpha \in[0,1]$ be the share of abatement capital permitted in the rate base. Given these definitions, the ROR constraint may be written as

$$
\frac{R\left(\mathbf{x}_{M+1}\right)-\sum_{m=1}^{M} w_{m} x_{m}}{x_{M+1}+\alpha x_{M+2}} \leq s,
$$

and states that the ROR on the sum of the regulated input and fraction $\alpha$ of the abatement capital permitted in the rate base does not exceed the highest ROR permitted by the regulating agency. It differs from that of Averch and Johnson (1962) by the $\alpha x_{M+2}$ term in the denominator. Though this difference is seemingly trivial, it, in conjunction with the CAC pollution con- 
straint, profoundly changes many of the basic properties of the standard A-J model, including the form of its intrinsic comparative statics, as will be seen in Sections 2 and 3, respectively.

To complete the model, we incorporate a CAC pollution constraint. Let $h(\cdot) \in C^{(2)}$ be the emissions function of the firm, with $h_{y}\left(y, x_{M+2}\right)>0$ and $h_{x_{M+2}}\left(y, x_{M+2}\right)<0$. Then the fixedlevel-of-emissions constraint is $h\left(f\left(\mathbf{x}_{M+1}\right), x_{M+2}\right) \leq \bar{E}$, where $\bar{E}$ is the highest level of emissions permitted. The fixed-level-of-emissions constraint is one of the six commonly implemented constraints identified by Helfand (1991) and noted earlier. The other constraints will be considered in Section 5.

Finally, with parameter vector $\mathbf{b} \stackrel{\text { def }}{=}\left(\mathbf{w}_{M+2}, s, \alpha, \bar{E}\right) \in \mathbb{R}_{++}^{M+5}$, the monopolist is asserted to solve the following constrained optimization problem:

$$
\begin{gathered}
\pi^{*}(\mathbf{b}) \stackrel{\text { def }}{=} \max _{\mathbf{x}_{M+2}}\left\{R\left(\mathbf{x}_{M+1}\right)-\sum_{m=1}^{M+2} w_{m} x_{m}\right\} \\
\text { s.t. } R\left(\mathbf{x}_{M+1}\right)-\sum_{m=1}^{M} w_{m} x_{m} \leq s\left[x_{M+1}+\alpha x_{M+2}\right], h\left(f\left(\mathbf{x}_{M+1}\right), x_{M+2}\right) \leq \bar{E} .
\end{gathered}
$$

Hereafter, we refer to the constrained optimization problem (2) as the augmented A-J model. In contrast, the generalized A-J model of Caputo and Partovi $(2002,2008)$ is given by

$$
\max _{\mathbf{x}_{M+1}}\left\{R\left(\mathbf{x}_{M+1}\right)-\sum_{m=1}^{M+1} w_{m} x_{m} \text { s.t. } R\left(\mathbf{x}_{M+1}\right)-\sum_{m=1}^{M} w_{m} x_{m} \leq s x_{M+1}\right\} \text {. }
$$

Henceforth, we refer to the constrained optimization problem (3) as the standard A-J model. It is generated from problem (2) by deleting the CAC constraint and setting $\alpha \equiv 0$ and $x_{M+2} \equiv 0 .^{3}$

The following assumptions, parallel to those of Caputo and Partovi (2008), are maintained throughout the paper:

\footnotetext{
3 In passing, note that Rose et al. (1992) committed an algebraic error in writing the ROR constraint. In our notation, the constraint of Rose et al. (1992) would be written as$$
R\left(\mathbf{x}_{M+1}\right)-\sum_{m=1}^{M} w_{m} x_{m} \leq s x_{M+1}+\left[\alpha s+(1-\alpha) w_{M+2}\right] x_{M+2}
$$

The error is the inclusion of the term $(1-\alpha) w_{M+2} x_{M+2}$ on the right-hand side of the preceding equation. As $\alpha$ was close to 1 at the time of their study, it seems unlikely that the error had serious practical implications for their work.
} 
(A.1) $s>w_{M+1}$.

(A.2) $s>w_{M+2} / \alpha$.

(A.3) For each $\mathbf{b} \stackrel{\text { def }}{=}\left(\mathbf{w}_{M+2}, s, \alpha, \bar{E}\right) \in P^{\text {open }} \subset \mathbb{R}_{++}^{M+5}$, there exists an interior locally $C^{(1)}$ solution to problem (2), denoted by $\mathbf{x}_{M+2}=\mathbf{x}_{M+2}^{*}(\mathbf{b})$, with $\lambda_{1}=\lambda_{1}^{*}(\mathbf{b})$ and $\lambda_{2}=\lambda_{2}^{*}(\mathbf{b})$ representing the optimal values of the Lagrange multipliers corresponding to the ROR and CAC constraints, respectively.

(A.4) The ROR and CAC constraints are binding, and not just binding, at $\mathbf{x}_{M+2}=\mathbf{x}_{M+2}^{*}(\mathbf{b})$.

Together, assumptions (A.1) and (A.2) permit the monopolist to make positive profit. Supposition (A.3) guarantees the existence of a locally continuously differentiable solution, enabling differentiable comparative statics analysis to be performed. Because both the ROR and CAC constraints are binding at the solution by assumption (A.4), the solution of the augmented A-J model does not coincide with that of the standard A-J model. This supposition is therefore crucial for studying our model, as we wish to investigate the consequences of both constraints on the firm's behavior. Assumptions (A.1), (A.3), and (A.4) are essentially identical to those employed in the standard A-J model of Caputo and Partovi (2008), whereas assumption (A.2) is unique to our model.

Next we obtain the first-order necessary conditions. To this end, define the Lagrangian of problem (2) is defined as

$$
\begin{aligned}
L\left(\mathbf{x}_{M+2}, \lambda_{1}, \lambda_{2} ; \mathbf{b}\right) \stackrel{\text { def }}{=} R\left(\mathbf{x}_{M+1}\right)-\sum_{m=1}^{M+2} w_{m} x_{m} & +\lambda_{1}\left[\sum_{m=1}^{M} w_{m} x_{m}-R\left(\mathbf{x}_{M+1}\right)+s x_{M+1}+\alpha s x_{M+2}\right] \\
& +\lambda_{2}\left[\bar{E}-h\left(f\left(\mathbf{x}_{M+1}\right), x_{M+2}\right)\right] .
\end{aligned}
$$

Assuming that the nondegenerate constraint qualification holds at the solution, Theorem 2.3 of Takayama (1993) asserts that the first-order necessary conditions of the augmented A-J model are given by

$$
L_{x_{l}}=\left[1-\lambda_{1}\right]\left[R_{x_{l}}\left(\mathbf{x}_{M+1}\right)-w_{l}\right]-\lambda_{2} h_{y}\left(y, x_{M+2}\right) f_{x_{l}}\left(\mathbf{x}_{M+1}\right)=0, l=1,2, \ldots, M,
$$




$$
\begin{gathered}
L_{x_{M+1}}=R_{x_{M+1}}\left(\mathbf{x}_{M+1}\right)-w_{M+1}+\lambda_{1}\left[s-R_{x_{M+1}}\left(\mathbf{x}_{M+1}\right)\right]-\lambda_{2} h_{y}\left(y, x_{M+2}\right) f_{x_{M+1}}\left(\mathbf{x}_{M+1}\right)=0, \\
L_{x_{M+2}}=-w_{M+2}+\lambda_{1} \alpha s-\lambda_{2} h_{x_{M+2}}\left(y, x_{M+2}\right)=0, \\
L_{\lambda_{1}}=\sum_{m=1}^{M} w_{m} x_{m}-R\left(\mathbf{x}_{M+1}\right)+s x_{M+1}+\alpha s x_{M+2} \geq 0, \lambda_{1} \geq 0, L_{\lambda_{1}} \cdot \lambda_{1}=0, \\
L_{\lambda_{2}}=\bar{E}-h\left(y, x_{M+2}\right) \geq 0, \lambda_{2} \geq 0, L_{\lambda_{2}} \cdot \lambda_{2}=0,
\end{gathered}
$$

where $y=f\left(\mathbf{x}_{M+1}\right)$. Using the first-order necessary conditions for the corresponding standard A-J model, Caputo and Partovi $(2002,2008)$ deduce several conclusions about its solution and made a distinction between the ranges of possible values of the Lagrange multiplier associated with the ROR constraint. We now proceed to show that several of their results no longer hold in the augmented A-J model using the preceding first-order necessary conditions.

To begin, note that Caputo and Partovi (2002, Lemma 1) showed that in the standard A-J model the Lagrange multiplier associated with the ROR constraint is positive and not equal to one, but was otherwise unrestricted. In the augmented A-J model, however, more can be said about the Lagrange multiplier associated with the ROR constraint. First, note that supposition (A.4) and Eqs. (8) and (9) imply that $\lambda_{1}^{*}(\mathbf{b})>0$ and $\lambda_{2}^{*}(\mathbf{b})>0$. Moreover, because $\lambda_{2}^{*}(\mathbf{b})>0$ and $h_{x_{M+2}}\left(y, x_{M+2}\right)<0$, it follows from Eq. (7) that $\lambda_{1}^{*}(\mathbf{b})<w_{M+2} / \alpha s<1$, the last inequality following from assumption (A.2). Combining the preceding results for $\lambda_{1}^{*}(\mathbf{b})$ permits us to conclude that $\lambda_{1}^{*}(\mathbf{b}) \in(0,1)$, thereby establishing the following basic result in the augmented A-J model.

Lemma 1. Under assumptions (A.1)-(A.4), the value of the Lagrange multiplier corresponding to the ROR constraint in the augmented A-J model defined by Eq. (2) satisfies $\lambda_{1}^{*}(\mathbf{b}) \in(0,1)$.

The permissible range of $\lambda_{1}^{*}(\mathbf{b})$ indicated by Lemma 1 lies in the region referred to by Caputo and Partovi (2008, p. 373) as "normal," because in the standard A-J model, $\lambda_{1}^{*}(\mathbf{b}) \in(0,1)$ is equivalent to the existence of the normal A-J effect by Lemma 2 of Caputo and Partovi (2002), i.e., $\lambda_{1}^{*}(\mathbf{b}) \in(0,1) \Leftrightarrow \partial x_{M+1}^{*}(\mathbf{b}) / \partial s<0$ in the standard A-J model. Furthermore, Lemma 1 shows 
that the so-called "anomalous" case, namely $\lambda_{1}^{*}(\mathbf{b})>1$, does not arise in the augmented A-J model, in contrast to the standard A-J model.

Given that $\lambda_{2}^{*}(\mathbf{b})>0, h_{y}\left(y, x_{M+2}\right)>0$, and $f_{x_{l}}\left(\mathbf{x}_{M+1}\right)>0$, using Lemma 1 in Eq. (5) implies that $R_{x_{l}}\left(\mathbf{x}_{M+1}\right)>w_{l}, l=1,2, \ldots, M$. Hence the marginal revenue of every unregulated input is greater than its marginal cost at the optimum. This conclusion contrasts sharply with that reached by Caputo and Partovi [2002, Eq. (7)] in the standard A-J model, where they showed that $R_{x_{l}}\left(\mathbf{x}_{M+1}\right)=w_{l}$ holds for all unregulated inputs. The reason for the differing conclusions is the presence of the binding CAC pollution constraint. To see this, assume that the CAC constraint is not binding at the solution, which implies that $\lambda_{2}^{*}(\mathbf{b}) \equiv 0$ by Eq. (9). Then upon applying $\lambda_{2}^{*}(\mathbf{b}) \equiv 0$ and Lemma 1 to Eq. (5) we arrive at $R_{x_{l}}\left(\mathbf{x}_{M+1}\right)=w_{l}$ for all unregulated inputs, just as Caputo and Partovi [2002, Eq. (7)] found.

The result that $R_{x_{l}}\left(\mathbf{x}_{M+1}\right)>w_{l}, l=1,2, \ldots, M$, in the augmented A-J model has an intuitive explanation. Because the emissions constraint is binding, the firm would prefer to pollute more than it is permitted. Seeing as the marginal product of the unregulated inputs are positive and that the emissions rate is increasing in output, it follows that the emissions rate is an increasing function of the unregulated inputs. Hence one way for the firm to reduce its emissions rate below the unregulated rate (and thus satisfy the CAC emissions constraint) is to reduce its use of the unregulated inputs. Given a downward sloping marginal revenue product curve for $x_{l}$, the marginal condition $R_{x_{l}}\left(\mathbf{x}_{M+1}\right)>w_{l}$ is fully consistent with the above explanation in that it asserts that $x_{l}$ is "underemployed," ceteris paribus, compared to the situation in which the CAC emissions constraint is not binding.

Another property of the standard A-J model does not necessarily hold in the augmented A-J model, as we now demonstrate. In the standard A-J model, Caputo and Partovi (2008, p. 378) showed that in the normal case, i.e., $\lambda_{1}^{*}(\mathbf{b}) \in(0,1)$, the inequality $R_{x_{M+1}}\left(\mathbf{x}_{M+1}\right)<w_{M+1}$ holds. This result does not necessarily hold in the augmented A-J model, as can be seen by inspection of Eq. (6). The presence of the binding environmental constraint is again the reason for this conclusion. The reader is referred to Caputo and Partovi (2008, p. 378) for the economic intuition 
behind the marginal condition $R_{x_{M+1}}\left(\mathbf{x}_{M+1}\right)<w_{M+1}$ and what it implies. In a sense, the intuition is the opposite of that given in the preceding paragraph for $R_{x_{l}}\left(\mathbf{x}_{M+1}\right)>w_{l}$.

A further difference between the standard and augmented A-J models concerns the A-J effect, that is, the sign of the comparative statics expression $\partial x_{M+1}^{*}(\mathbf{b}) / \partial s$. Among other things, Lemma 2 of Caputo and Partovi (2002) establishes the equivalency of $\lambda_{1}^{*}(\mathbf{b}) \in(0,1)$ and $\partial x_{M+1}^{*}(\mathbf{b}) / \partial s<0$ in the standard A-J model, the latter being the normal A-J effect. No such equivalency exists in the augmented A-J model. To see this, use the fact that $\lambda_{1}^{*}(\mathbf{b}) \in(0,1)$ to write Eq. (8) as an identity, namely, $\sum_{m=1}^{M} w_{m} x_{m}^{*}(\mathbf{b})-R\left(\mathbf{x}_{M+1}^{*}(\mathbf{b})\right)+s x_{M+1}^{*}(\mathbf{b})+\alpha s x_{M+2}^{*}(\mathbf{b}) \equiv 0$, and then differentiate the identity with respect to $s$ to get

$$
\begin{aligned}
\sum_{m=1}^{M}\left[w_{m}-R_{x_{m}}\left(\mathbf{x}_{M+1}^{*}(\mathbf{b})\right)\right] \frac{\partial x_{m}^{*}(\mathbf{b})}{\partial s} & +\left[s-R_{x_{M+1}}\left(\mathbf{x}_{M+1}^{*}(\mathbf{b})\right)\right] \frac{\partial x_{M+1}^{*}(\mathbf{b})}{\partial s}+x_{M+1}^{*}(\mathbf{b}) \\
& +\alpha s \frac{\partial x_{M+2}^{*}(\mathbf{b})}{\partial s}+\alpha x_{M+2}^{*}(\mathbf{b}) \equiv 0
\end{aligned}
$$

In the standard A-J model, $\alpha \equiv 0$ and $x_{M+2}^{*}(\mathbf{b})$. Combining these facts with the necessary conditions $R_{x_{l}}\left(\mathbf{x}_{M+1}\right)=w_{l}$ for all of the unregulated inputs and a much simpler expression for $\lambda_{1}^{*}(\mathbf{b})$ in the standard A-J model leads to a vast simplification of the comparative statics expression for $\partial x_{M+1}^{*}(\mathbf{b}) / \partial s$ and the aforesaid equivalency. On the other hand, use of the first-order necessary conditions given in Eqs. (5)-(9) does not result in such a simplification of the preceding expression in the augmented A-J model. Accordingly, and in general, the equivalency between $\lambda_{1}^{*}(\mathbf{b}) \in(0,1)$ and $\partial x_{M+1}^{*}(\mathbf{b}) / \partial s<0$ fails to hold in the augmented A-J model. As before, the binding CAC constraint is responsible for the difference.

The final discrepancy between the standard and augmented A-J models to be discussed at this juncture concerns the price of the regulated input. Caputo and Partovi (2008, p. 372) showed that all of the factor demand functions in the standard A-J model are independent of the price of the regulated input. As can be seen by inspection of Eqs. (5)-(9), the same does not hold, in general, in the augmented A-J model. The binding CAC constraint is again the reason for the differing conclusions. 
Summing up, the presence of the binding CAC pollution constraint causes substantial changes in the standard A-J model, so much so that few of the basic properties of the standard A$\mathrm{J}$ model continue to hold in the augmented A-J model. These differences demonstrate that the standard and augmented A-J models fundamentally differ from one another, i.e., that the binding CAC pollution constraint changes the behavior of the firm in basic ways. In the next section we show how the fundamental comparative statics properties of the model are affected by the introduction of the said constraint.

\section{Comparative Statics Results}

The central result of our paper is stated in the ensuing theorem, and an economic interpretation of its content follows. Its proof may be found in the Appendix.

Theorem 1. Under assumptions (A.1)-(A.4), the comparative statics of model (2) are contained in the following $(M+3) \times(M+3)$ negative semidefinite constraint-free matrix:

$$
\Omega^{*}(\mathbf{b}) \stackrel{\text { def }}{=}\left[\begin{array}{cccc}
\Omega_{\sigma, \tau} & \Omega_{\sigma,(M+1)} & \Omega_{\sigma,(M+2)} & \Omega_{\sigma,(M+3)} \\
\Omega_{(M+1), \tau} & \Omega_{(M+1),(M+1)} & \Omega_{(M+1),(M+2)} & \Omega_{(M+1),(M+3)} \\
\Omega_{(M+2), \tau} & \Omega_{(M+2),(M+1)} & \Omega_{(M+2),(M+2)} & \Omega_{(M+2),(M+3)} \\
\Omega_{(M+3), \tau} & \Omega_{(M+3),(M+1)} & \Omega_{(M+3),(M+2)} & \Omega_{(M+3),(M+3)}
\end{array}\right],
$$

where $\sigma, \tau=1,2, \ldots, M$, and

$$
\begin{aligned}
\Omega_{\sigma, \tau}= & {\left[1-\lambda_{1}^{*}(\mathbf{b})\right]\left[\frac{\partial x_{\sigma}^{*}(\mathbf{b})}{\partial w_{\tau}}-\frac{x_{\tau}^{*}(\mathbf{b})}{x_{M+1}^{*}(\mathbf{b})+\alpha x_{M+2}^{*}(\mathbf{b})} \frac{\partial x_{\sigma}^{*}(\mathbf{b})}{\partial s}\right] } \\
& +\frac{\lambda_{1}^{*}(\mathbf{b}) x_{\sigma}^{*}(\mathbf{b})}{x_{M+1}^{*}(\mathbf{b})+\alpha x_{M+2}^{*}(\mathbf{b})}\left[\frac{\partial x_{M+1}^{*}(\mathbf{b})}{\partial w_{\tau}}-\frac{x_{\tau}^{*}(\mathbf{b})}{x_{M+1}^{*}(\mathbf{b})+\alpha x_{M+2}^{*}(\mathbf{b})} \frac{\partial x_{M+1}^{*}(\mathbf{b})}{\partial s}\right] \\
& +\frac{\alpha \lambda_{1}^{*}(\mathbf{b}) x_{\sigma}^{*}(\mathbf{b})}{x_{M+1}^{*}(\mathbf{b})+\alpha x_{M+2}^{*}(\mathbf{b})}\left[\frac{\partial x_{M+2}^{*}(\mathbf{b})}{\partial w_{\tau}}-\frac{x_{\tau}^{*}(\mathbf{b})}{x_{M+1}^{*}(\mathbf{b})+\alpha x_{M+2}^{*}(\mathbf{b})} \frac{\partial x_{M+2}^{*}(\mathbf{b})}{\partial s}\right], \\
\Omega_{\sigma,(M+1)}= & {\left[1-\lambda_{1}^{*}(\mathbf{b})\right] \frac{\partial x_{\sigma}^{*}(\mathbf{b})}{\partial w_{M+1}}+\frac{\lambda_{1}^{*}(\mathbf{b}) x_{\sigma}^{*}(\mathbf{b})}{x_{M+1}^{*}(\mathbf{b})+\alpha x_{M+2}^{*}(\mathbf{b})}\left[\frac{\partial x_{M+1}^{*}(\mathbf{b})}{\partial w_{M+1}}+\alpha \frac{\partial x_{M+2}^{*}(\mathbf{b})}{\partial w_{M+1}}\right], }
\end{aligned}
$$




$$
\begin{aligned}
& \Omega_{\sigma,(M+2)}(\mathbf{b})=\left[1-\lambda_{1}^{*}(\mathbf{b})\right] \frac{\partial x_{\sigma}^{*}(\mathbf{b})}{\partial w_{M+2}}+\frac{\lambda_{1}^{*}(\mathbf{b}) x_{\sigma}^{*}(\mathbf{b})}{x_{M+1}^{*}(\mathbf{b})+\alpha x_{M+2}^{*}(\mathbf{b})}\left[\frac{\partial x_{M+1}^{*}(\mathbf{b})}{\partial w_{M+2}}+\alpha \frac{\partial x_{M+2}^{*}(\mathbf{b})}{\partial w_{M+2}}\right], \\
& \Omega_{\sigma,(M+3)}(\mathbf{b})=\left[1-\lambda_{1}^{*}(\mathbf{b})\right]\left[\frac{\partial x_{\sigma}^{*}(\mathbf{b})}{\partial \alpha}-\frac{s x_{M+2}^{*}(\mathbf{b})}{x_{M+1}^{*}(\mathbf{b})+\alpha x_{M+2}^{*}(\mathbf{b})} \frac{\partial x_{\sigma}^{*}(\mathbf{b})}{\partial s}\right] \\
& +\frac{\lambda_{1}^{*}(\mathbf{b}) x_{\sigma}^{*}(\mathbf{b})}{x_{M+1}^{*}(\mathbf{b})+\alpha x_{M+2}^{*}(\mathbf{b})}\left[\frac{\partial x_{M+1}^{*}(\mathbf{b})}{\partial \alpha}-\frac{s x_{M+2}^{*}(\mathbf{b})}{x_{M+1}^{*}(\mathbf{b})+\alpha x_{M+2}^{*}(\mathbf{b})} \frac{\partial x_{M+1}^{*}(\mathbf{b})}{\partial s}\right] \\
& +\frac{\alpha \lambda_{1}^{*}(\mathbf{b}) x_{\sigma}^{*}(\mathbf{b})}{x_{M+1}^{*}(\mathbf{b})+\alpha x_{M+2}^{*}(\mathbf{b})}\left[\frac{\partial x_{M+2}^{*}(\mathbf{b})}{\partial \alpha}-\frac{s x_{M+2}^{*}(\mathbf{b})}{x_{M+1}^{*}(\mathbf{b})+\alpha x_{M+2}^{*}(\mathbf{b})} \frac{\partial x_{M+2}^{*}(\mathbf{b})}{\partial s}\right] \text {, } \\
& \Omega_{(M+1), \tau}(\mathbf{b})=\frac{\partial x_{M+1}^{*}(\mathbf{b})}{\partial w_{\tau}}-\frac{x_{\tau}^{*}(\mathbf{b})}{x_{M+1}^{*}(\mathbf{b})+\alpha x_{M+2}^{*}(\mathbf{b})} \frac{\partial x_{M+1}^{*}(\mathbf{b})}{\partial s}, \\
& \Omega_{(M+1),(M+1)}(\mathbf{b})=\frac{\partial x_{M+1}^{*}(\mathbf{b})}{\partial w_{M+1}}, \\
& \Omega_{(M+1),(M+2)}(\mathbf{b})=\frac{\partial x_{M+1}^{*}(\mathbf{b})}{\partial w_{M+2}}, \\
& \Omega_{(M+1),(M+3)}(\mathbf{b})=\frac{\partial x_{M+1}^{*}(\mathbf{b})}{\partial \alpha}-\frac{s x_{M+2}^{*}(\mathbf{b})}{x_{M+1}^{*}(\mathbf{b})+\alpha x_{M+2}^{*}(\mathbf{b})} \frac{\partial x_{M+1}^{*}(\mathbf{b})}{\partial s}, \\
& \Omega_{(M+2), \tau}(\mathbf{b})=\frac{\partial x_{M+2}^{*}(\mathbf{b})}{\partial w_{\tau}}-\frac{x_{\tau}^{*}(\mathbf{b})}{x_{M+1}^{*}(\mathbf{b})+\alpha x_{M+2}^{*}(\mathbf{b})} \frac{\partial x_{M+2}^{*}(\mathbf{b})}{\partial s}, \\
& \Omega_{(M+2),(M+1)}(\mathbf{b})=\frac{\partial x_{M+2}^{*}(\mathbf{b})}{\partial w_{M+1}}, \\
& \Omega_{(M+2),(M+2)}(\mathbf{b})=\frac{\partial x_{M+2}^{*}(\mathbf{b})}{\partial w_{M+2}}, \\
& \Omega_{(M+2),(M+3)}(\mathbf{b})=\frac{\partial x_{M+2}^{*}(\mathbf{b})}{\partial \alpha}-\frac{s x_{M+2}^{*}(\mathbf{b})}{x_{M+1}^{*}(\mathbf{b})+\alpha x_{M+2}^{*}(\mathbf{b})} \frac{\partial x_{M+2}^{*}(\mathbf{b})}{\partial s}, \\
& \Omega_{(M+3), \tau}(\mathbf{b})=\frac{s \lambda_{1}^{*}(\mathbf{b}) x_{M+2}^{*}(\mathbf{b})}{x_{M+1}^{*}(\mathbf{b})+\alpha x_{M+2}^{*}(\mathbf{b})}\left[\frac{\partial x_{M+1}^{*}(\mathbf{b})}{\partial w_{\tau}}-\frac{x_{\tau}^{*}(\mathbf{b})}{x_{M+1}^{*}(\mathbf{b})+\alpha x_{M+2}^{*}(\mathbf{b})} \frac{\partial x_{M+1}^{*}(\mathbf{b})}{\partial s}\right] \\
& -\frac{s \lambda_{1}^{*}(\mathbf{b}) x_{M+1}^{*}(\mathbf{b})}{x_{M+1}^{*}(\mathbf{b})+\alpha x_{M+2}^{*}(\mathbf{b})}\left[\frac{\partial x_{M+2}^{*}(\mathbf{b})}{\partial w_{\tau}}-\frac{x_{\tau}^{*}(\mathbf{b})}{x_{M+1}^{*}(\mathbf{b})+\alpha x_{M+2}^{*}(\mathbf{b})} \frac{\partial x_{M+2}^{*}(\mathbf{b})}{\partial s}\right] \text {, }
\end{aligned}
$$




$$
\begin{gathered}
\Omega_{(M+3),(M+1)}(\mathbf{b})=\frac{s \lambda_{1}^{*}(\mathbf{b})}{x_{M+1}^{*}(\mathbf{b})+\alpha x_{M+2}^{*}(\mathbf{b})}\left[x_{M+2}^{*}(\mathbf{b}) \frac{\partial x_{M+1}^{*}(\mathbf{b})}{\partial w_{M+1}}-x_{M+1}^{*}(\mathbf{b}) \frac{\partial x_{M+2}^{*}(\mathbf{b})}{\partial w_{M+1}}\right], \\
\Omega_{(M+3),(M+2)}(\mathbf{b})=\frac{s \lambda_{1}^{*}(\mathbf{b})}{x_{M+1}^{*}(\mathbf{b})+\alpha x_{M+2}^{*}(\mathbf{b})}\left[x_{M+2}^{*}(\mathbf{b}) \frac{\partial x_{M+1}^{*}(\mathbf{b})}{\partial w_{M+2}}-x_{M+1}^{*}(\mathbf{b}) \frac{\partial x_{M+2}^{*}(\mathbf{b})}{\partial w_{M+2}}\right], \\
\Omega_{(M+3),(M+3)}(\mathbf{b})= \\
\frac{s \lambda_{1}^{*}(\mathbf{b}) x_{M+2}^{*}(\mathbf{b})}{x_{M+1}^{*}(\mathbf{b})+\alpha x_{M+2}^{*}(\mathbf{b})}\left[\frac{\partial x_{M+1}^{*}(\mathbf{b})}{\partial \alpha}-\frac{s x_{M+2}^{*}(\mathbf{b})}{x_{M+1}^{*}(\mathbf{b})+\alpha x_{M+2}^{*}(\mathbf{b})} \frac{\partial x_{M+1}^{*}(\mathbf{b})}{\partial s}\right] \\
-\frac{s \lambda_{1}^{*}(\mathbf{b}) x_{M+1}^{*}(\mathbf{b})}{x_{M+1}^{*}(\mathbf{b})+\alpha x_{M+2}^{*}(\mathbf{b})}\left[\frac{\partial x_{M+2}^{*}(\mathbf{b})}{\partial \alpha}-\frac{s x_{M+2}^{*}(\mathbf{b})}{x_{M+1}^{*}(\mathbf{b})+\alpha x_{M+2}^{*}(\mathbf{b})} \frac{\partial x_{M+2}^{*}(\mathbf{b})}{\partial s}\right] .
\end{gathered}
$$

Theorem 1 gives the intrinsic comparative statics of the augmented A-J model, and is therefore the equivalent of the Slutsky matrix of neoclassical consumer theory. It is more complex than the prototype Slutsky matrix and its counterpart derived from the standard A-J model as given by Theorem 1 of Caputo and Partovi (2008). This is a result of the fact that the augmented A-J model is itself more complex than the neoclassical utility maximization model and the standard A-J model. Note, however, the preservation of the Slutsky-like form of the comparative statics expressions, i.e., the compensated form of the comparative statics expressions, confirming the basic role they play in the derivation of refutable hypotheses in economic models.

Because the comparative statics matrix $\Omega^{*}(\mathbf{b})$ is negative semidefinite, its diagonal elements are nonpositive, implying that $\partial x_{M+1}^{*} / \partial w_{M+1} \leq 0$ and $\partial x_{M+2}^{*} / \partial w_{M+2} \leq 0$, i.e., the regulated input and abatement capital factor demand functions are not upward-sloping in their own prices. These are the only single-term partial derivative comparative statics expressions that can be signed. The reason for this is that the prices $w_{M+1}$ and $w_{M+2}$ enter only the objective function, whereas all the other parameters appear in one of the constraints. Because of this structural feature, it can be shown that $\pi^{*}(\cdot)$ is locally convex in $\left(w_{M+1}, w_{M+2}\right)$, which in conjunction with the envelope results in Proposition 1, can be used to derive the two preceding comparative statics results. The same does not follow for the other parameters of the model seeing as they enter one of the constraints. Consequently, all the other intrinsic comparative statics expressions are of a gen- 
generalized Slutsky form, consisting of linear combinations of partial derivatives, i.e., they are compensated comparative statics forms. As stated above, this is due to the fact that the said parameters enter one of the constraints.

As an example of the above, observe that $\Omega_{(M+3),(M+3)}(\mathbf{b}) \leq 0$ is equivalent to

$$
\begin{aligned}
x_{M+2}^{*}(\mathbf{b})\left[\frac{\partial x_{M+1}^{*}(\mathbf{b})}{\partial \alpha}-\frac{s x_{M+2}^{*}(\mathbf{b})}{x_{M+1}^{*}(\mathbf{b})+\alpha x_{M+2}^{*}(\mathbf{b})} \frac{\partial x_{M+1}^{*}(\mathbf{b})}{\partial s}\right] \\
\quad-x_{M+1}^{*}(\mathbf{b})\left[\frac{\partial x_{M+2}^{*}(\mathbf{b})}{\partial \alpha}-\frac{s x_{M+2}^{*}(\mathbf{b})}{x_{M+1}^{*}(\mathbf{b})+\alpha x_{M+2}^{*}(\mathbf{b})} \frac{\partial x_{M+2}^{*}(\mathbf{b})}{\partial s}\right] \leq 0
\end{aligned}
$$

because $\mathbf{x}_{M+2}^{*}(\mathbf{b}) \in \mathbb{R}_{++}^{M+2}$ by assumption (A.3), $\lambda_{1}^{*}(\mathbf{b}) \in(0,1)$ by Lemma 1 , and $s \in \mathbb{R}_{++}$. While Eq. (27) may not appear to have an obvious economic interpretation, it can be readily shown that it is equivalent to

$$
\left[\frac{\partial}{\partial \alpha}-\left[\frac{s x_{M+2}^{*}(\mathbf{b})}{x_{M+1}^{*}(\mathbf{b})+\alpha x_{M+2}^{*}(\mathbf{b})}\right] \frac{\partial}{\partial s}\right] \circ\left[\frac{x_{M+1}^{*}(\mathbf{b})}{x_{M+2}^{*}(\mathbf{b})}\right] \leq 0,
$$

which does. Equation (28) asserts that the demand for abatement capital increases relative to that for the regulated input, when a ROR-compensated increase in the share of abatement capital permitted in the rate base occurs. Thus the property $\Omega_{(M+3),(M+3)}(\mathbf{b}) \leq 0$ is nothing more than a compensated and relative law-of-demand-like property in the augmented A-J model.

The negative semidefiniteness of the comparative statics matrix implies its symmetry. By symmetry, $\Omega_{(M+1),(M+2)}(\mathbf{b})=\Omega_{(M+2),(M+1)}(\mathbf{b})$, which amounts to the simple reciprocity condition $\partial x_{M+1}^{*} / \partial w_{M+2}=\partial x_{M+2}^{*} / \partial w_{M+1}$. All of the other reciprocity results involve compensated comparative statics expressions, because, as remarked above, the parameters involved appear in one of the constraints.

An important difference in the intrinsic comparative statics properties of the two models is the role played by the archetype A-J comparative statics expression $\partial x_{M+1}^{*} / \partial s$. In the standard A-J model, Theorem 1 of Caputo and Partovi (2008) demonstrates that this expression plays a fundamental role in the form and economic interpretation of the intrinsic comparative statics of the model. In our augmented A-J model, on the other hand, Theorem 1 shows that no such simi- 
lar role is played by $\partial x_{M+1}^{*} / \partial s$. In addition, the intrinsic comparative statics properties of the standard A-J model depend crucially on whether $\lambda_{1}^{*}(\mathbf{b}) \in(0,1)$ or $\lambda_{1}^{*}(\mathbf{b})>1$, as shown by Caputo and Partovi (2002, Lemma 2), whereas the same is not true of the augmented A-J model, as the latter range is ruled out by Lemma 1 . In spite of these differences, the fact remains that, in general, $\partial x_{M+1}^{*} / \partial s$ may be positive or negative in either model.

Even though the comparative statics matrix $\Omega^{*}(\mathbf{b})$ is more complicated than the Slutsky matrix of neoclassical consumer theory or its counterpart from the standard A-J model, its entries are all observable. As a result, an empirical test of its negative semidefiniteness can be carried out, as we now demonstrate. Recall that the parameter vector for the augmented A-J model is given by $\mathbf{b} \stackrel{\text { def }}{=}\left(\mathbf{w}_{M+2}, s, \alpha, \bar{E}\right)$. The data on $\mathbf{w}_{M+2}$, the competitive prices of the factors of production and abatement capital, is generally available, as is the data on the input vector $\mathbf{x}_{M+2}$. The fraction of abatement capital allowed in the rate base, $\alpha$, the fair rate of return on capital, $s$, and $\bar{E}$, the upper bound on the emission rate, can be obtained from the regulating agencies. Thus one can estimate the $M+2$ factor demands $\mathbf{x}_{M+2}=\mathbf{x}_{M+2}^{*}(\mathbf{b})$ and derive the necessary partial derivatives that make up the elements of $\Omega^{*}(\mathbf{b})$. In order to test the negative semidefiniteness of $\Omega^{*}(\mathbf{b})$, an empirical estimate of $\lambda_{1}=\lambda_{1}^{*}(\mathbf{b})$ is also required, as can be seen by inspection of Theorem 1 . It can be obtained from the above data by estimating the indirect profit function $\pi^{*}(\cdot)$ and using the envelope results contained in Proposition 1 in the ensuing section. For example, making use of Eq. (30), (31), and (33), or Eqs. (31) and (32), one may obtain an estimate of $\lambda_{1}^{*}(\mathbf{b})$. With this information, matrix $\Omega^{*}(\mathbf{b})$ can be computed and its negative semidefiniteness tested.

\section{Envelope Results}

A direct application of the archetypal envelope theorem to problem (2) yields the ensuing result.

Proposition 1. The envelope results of the augmented A-J model are given by

$$
\frac{\partial \pi^{*}(\mathbf{b})}{\partial w_{l}}=\left[\lambda_{1}^{*}(\mathbf{b})-1\right] x_{l}^{*}(\mathbf{b})<0, l=1, \ldots, M,
$$




$$
\begin{gathered}
\frac{\partial \pi^{*}(\mathbf{b})}{\partial w_{M+1}}=-x_{M+1}^{*}(\mathbf{b})<0, \\
\frac{\partial \pi^{*}(\mathbf{b})}{\partial w_{M+2}}=-x_{M+2}^{*}(\mathbf{b})<0, \\
\frac{\partial \pi^{*}(\mathbf{b})}{\partial \alpha}=s \lambda_{1}^{*}(\mathbf{b}) x_{M+2}^{*}(\mathbf{b})>0, \\
\frac{\partial \pi^{*}(\mathbf{b})}{\partial s}=\lambda_{1}^{*}(\mathbf{b})\left[x_{M+1}^{*}(\mathbf{b})+\alpha x_{M+2}^{*}(\mathbf{b})\right]>0, \\
\frac{\partial \pi^{*}(\mathbf{b})}{\partial \bar{E}}=\lambda_{2}^{*}(\mathbf{b})>0 .
\end{gathered}
$$

Equations (29) and (30) are identical in form to those of Caputo and Partovi (2008). As in the standard A-J model, $\partial \pi^{*}(\mathbf{b}) / \partial s$ is the product of the Lagrange multiplier of the ROR constraint with its denominator. Since parameters $w_{M+2}, \alpha$, and $\bar{E}$ are absent from the standard AJ model, there are no counterparts to Eqs. (31), (32), and (34). Equation (34) shows that $\lambda_{2}^{*}(\mathbf{b})$ is the shadow price of pollution, in that it represents the increase in the maximum profit of the monopolist when the upper bound on emissions is relaxed.

The envelope results in Eqs. (29)-(31) have an intuitive economic interpretation, namely, that the monopolist is worse off if the price of any input increases. This stands in contrast to the envelope results for the unregulated input prices in the standard A-J model, as Caputo and Partovi [2008, Eq. (20)] showed that an increase in the said prices decrease the firm's profit if and only if the value of the Lagrange multiplier lies in the open unit interval, i.e., the "normal" case prevails. Thus it is possible in the standard A-J model for an increase in the price of an unregulated input to increase a firm's profit, whereas that is not possible in the augmented A-J model by Lemma 1 and Eq. (29).

According to Eq. (32), the monopolist is better off if the share of abatement capital allowed in the rate base increases. The economic intuition behind the result is clear: if more abatement capital is allowed in the rate base, then the ROR constraint is less restrictive to the 
monopolist by Eq. (1), thus increasing profit. Similarly, according to Eq. (33), the monopolist is better off if the allowable ROR increases, as a higher ROR permits the monopolist to receive higher returns on its investments in the regulated input and abatement capital, and therefore higher profit. And finally, according to Eq. (34), the monopolist is better off if the upper bound on emissions increases. In this case the monopolist faces a less stringent pollution constraint, and thus the set of feasible input-output combinations is larger, thereby permitting higher profit.

\section{Other Types of CAC Pollution Constraints}

As Helfand (1991) documented, and as noted in Section 1, besides uniform restrictions on pollution emissions, there are five other types of CAC pollution constraints faced by monopolists. Note that the constraints below are taken from her work, but are adjusted to fit the specifics of our model. The proof of the ensuing proposition is relegated to the Appendix. A heuristic discussion of its veracity follows.

\section{Proposition 2. Consider the following CAC constraints:}

Type 1: Emissions per unit of output $h\left(y, x_{M+2}\right) / y \leq \bar{E}_{P F}$.

Type 2, case 1: Emissions per unit of regulated capital $h\left(y, x_{M+2}\right) / x_{M+1} \leq Z_{P I}$.

Type 2, case 2: Emissions per unit of abatement capital $h\left(y, x_{M+2}\right) / x_{M+2} \leq Z_{A I}$.

Type 3, case 1: Standard as a maximum amount of regulated capital $x_{M+1} \leq Z_{P I}^{\prime}$.

Type 3, case 2: Standard as a minimum amount of abatement capital $x_{M+2} \geq Z_{A I}^{\prime}$.

The comparative statics of problem (2) subject to the ROR constraint and any one of the above binding CAC constraints are identical in form to the comparative statics in Proposition 1.

It is important to understand precisely what is asserted by Proposition 2, as it is an unanticipated result. Proposition 2 does not assert that the solutions of the constrained maximization problem are the same regardless of which CAC constraint binds. In other words, and in general, the value of the factor demand functions differ depending on which of the six CAC constraints is binding. So in this sense the various $\mathrm{CAC}$ constraints do indeed have a differential impact on the 
optimal choices. On the other hand, Proposition 1 asserts that for each of the binding CAC constraints, the resulting comparative statics matrix has the same form as that in Theorem 1 . That is to say, the form of the intrinsic comparative statics is invariant to the type of CAC constraint imposed on the ROR regulated monopolist. In this sense the differing CAC constraints do not have a differential impact on how the firm qualitatively responds to parameter changes. Nonetheless, the values of the Slutsky-like combinations contained in the comparative statics matrix will differ, because the solutions of the constrained optimization problem differ depending on which CAC constraint is binding, as noted above.

As to why Proposition 2 holds, the following heuristic argument can be given. First observe that only one parameter enters each of the CAC constraints, and that this parameter does not enter the ROR constraint. Then note that the generalized compensated derivatives (GCDs) of problem (2), which are defined in the Appendix, are independent of $\bar{E}$, the parameter in the binding CAC constraint. Because of this and the fact that the GCDs are constructions in parameter space by definition, the exact manner in which the decision variables enter a CAC constraint is of no consequence for their form. Therefore, regardless of the type of CAC constraint imposed, as long as just one parameter is introduced in that constraint and the said parameter does not enter the ROR constraint, the resulting GCDs are identical in form to those derived in the Appendix for the emissions constraint, and are thus independent of the parameter in any of the

CAC constraints. As a result, when the said GCDs are applied to the first-order necessary conditions to derive the intrinsic comparative statics, they yield comparative statics expressions that are identical in form to those given in Theorem 1.

\section{Summary and Conclusions}

Decades after Averch and Johnson (1962) developed a model of a ROR regulated, profitmaximizing firm, Caputo and Partovi (2008) derived the model's intrinsic comparative statics properties. However, in addition to a ROR constraint, there exist firms that also must comply with environmental regulations. A model that takes this fact into account is needed to study the behavior of such firms. Although Rose et al. (1992) and Meran and Schwalbe (2006) developed 
such models, they did not explore their comparative statics properties much. We have filled in this gap in the literature by employing the formalism of Partovi and Caputo (2006) to obtain a semidefinite matrix containing the intrinsic comparative statics properties of the A-J model augmented by the inclusion of various forms of commonly implemented CAC emissions constraints.

Our analysis was structured around a comparison of the augmented and standard A-J models. We found few similarities and many differences between the two. In particular, because the ROR constraint is more intricate when abatement capital is allowed in the rate base, so too is the basic comparative statics matrix of the augmented A-J model. We also made a few unexpected discoveries, some technical and some substantive. As to the former, we demonstrated that the Lagrange multiplier associated with the ROR constraint in the augmented A-J model is restricted to lie in the open unit interval, a result that is not, in general, true for the standard A-J model. As examples of the latter, we found that (i) the A-J effect does not play an integral role in the augmented A-J model, and (ii) the intrinsic comparative statics properties of the augmented A-J model are invariant to the six types of CAC emissions constraints commonly implemented in practice.

In closing, two other points are worth mentioning again. One is that the comparative statics properties of the augmented A-J model are in principle observable, i.e., measurable, thereby implying that one can carry out a full empirical test of the model's intrinsic qualitative properties. The other is that the present model can serve as a yardstick for the development of related models that contemplate (i) price-cap regulation and CAC pollution constraints, (ii) ROR and market-based pollution constraints, and (iii) price-cap regulation and market-based pollution constraints. 


\section{Appendix}

Proof of Theorem 1. We adopt the terminology and follow the formalism of Partovi and Caputo (2006, pp. 36-38). The formalism can be viewed as having four main steps. First, obtain the vectors normal to the constraints in parameter space. Second, find a set of isovectors. Third, form the GCDs. And fourth, apply Theorem 1 of Partovi and Caputo $(2006,2007)$.

Step one begins by defining the two constraint functions $g^{1}(\cdot)$ and $g^{2}(\cdot)$ :

$$
\begin{gathered}
g^{1}\left(\mathbf{x}_{M+2} ; \mathbf{b}\right) \stackrel{\text { def }}{=} \sum_{m=1}^{M} w_{m} x_{m}-R\left(\mathbf{x}_{M+1}\right)+s x_{M+1}+\alpha s x_{M+2}, \\
g^{2}\left(\mathbf{x}_{M+2} ; \bar{E}\right) \stackrel{\text { def }}{=} \bar{E}-h\left(f\left(\mathbf{x}_{M+1}\right), x_{M+2}\right) .
\end{gathered}
$$

Since $\mathbf{b} \stackrel{\text { def }}{=}\left(\mathbf{w}_{M+2}, s, \alpha, \bar{E}\right) \in \mathbb{R}_{++}^{M+5}$, the gradient operator with respect to $\mathbf{b}$ is

$$
\nabla_{\mathbf{b}} \stackrel{\text { def }}{=}\left(\frac{\partial}{\partial w_{1}}, \frac{\partial}{\partial w_{2}}, \ldots, \frac{\partial}{\partial w_{M+2}}, \frac{\partial}{\partial s}, \frac{\partial}{\partial \alpha}, \frac{\partial}{\partial \bar{E}}\right)
$$

for the fixed-level-of-emissions constraint, and the corresponding 2-dimensional set of normal vectors in parameter space is

$$
\begin{aligned}
\nabla_{\mathbf{b}} g^{1}\left(\mathbf{x}_{M+2} ; \mathbf{b}\right)= & \left(x_{1}, \ldots, x_{M}, 0_{M+1}, 0_{M+2}, x_{M+1}+\alpha x_{M+2}, s x_{M+2}, 0_{M+5}\right), \\
& \nabla_{\mathbf{b}} g^{2}\left(\mathbf{x}_{M+2} ; \bar{E}\right)=\left(\mathbf{0}_{M+4}, 1_{M+5}\right),
\end{aligned}
$$

where $\mathbf{0}_{M+2}$ is the null vector in $\mathbb{R}^{M+2}$. Following the standard terminology adopted by Partovi and Caputo (2006), let the normal hyperplane be the vector space generated by the set of normal vectors in Eqs. (35) and (36). Seeing as $\nabla_{\mathbf{b}} g^{1}\left(\mathbf{x}_{M+2} ; \mathbf{b}\right) \cdot \nabla_{\mathbf{b}} g^{2}\left(\mathbf{x}_{M+2} ; \mathbf{b}\right)=0$, the two normal vectors are orthogonal, thus linearly independent. Therefore, the normal hyperplane generated by the two normal vectors is of two dimensions and the $2 \times(M+5)$ Jacobian matrix of the constraint functions evaluated at $\mathbf{b}=\overline{\mathbf{b}}$, namely, $\partial \mathbf{g}\left(\mathbf{x}_{M+2} ; \overline{\mathbf{b}}\right) / \partial \mathbf{b}$, is of rank 2. By the implicit function theorem, the dimension of the level surface of $\mathbf{g}(\cdot)$ passing through $\overline{\mathbf{b}}$ is $M+3$. The tangent hyperplane is generated by the vectors that are tangent to the level surface of $\mathbf{g}(\cdot)$ at $\overline{\mathbf{b}}$ and the said vectors point in the direction of no change of the constraint functions. Vectors that lie in the tangent hyperplane are called isovectors, and thus point in a null direction. Together, the 
normal and tangent vectors span every possible direction at point $\overline{\mathbf{b}}$ of the parameter space. Because the tangent hyperplane is of dimension $M+3$, we require $M+3$ linearly independent isovectors orthogonal to the normal hyperplane. Infinitely many sets of linearly independent isovectors satisfy the above criteria, with any valid choice leading to qualitatively equivalent comparative statics predictions.

The second step involves finding the isovectors. Let $\mathbf{e}_{T}^{S}$ be the standard basis vector, where $S$ is the position of the number 1 and $T$ is the dimension. We propose the following set of $M+3$ isovectors:

$$
\begin{gathered}
\mathbf{t}^{\sigma} \stackrel{\text { def }}{=}\left(\mathbf{e}_{M+2}^{\sigma},-\frac{x_{\sigma}}{x_{M+1}+\alpha x_{M+2}}, 0_{M+4}, 0_{M+5}\right), \sigma=1,2, \ldots, M, \\
\mathbf{t}^{M+1} \stackrel{\text { def }}{=} \mathbf{e}_{M+5}^{M+1}, \mathbf{t}^{M+2} \stackrel{\text { def }}{=} \mathbf{e}_{M+5}^{M+2}, \mathbf{t}^{M+3} \stackrel{\text { def }}{=}\left(\mathbf{0}_{M+2},-\frac{s x_{M+2}}{x_{M+1}+\alpha x_{M+2}}, 1_{M+4}, 0_{M+5}\right) .
\end{gathered}
$$

These vectors are perpendicular to both normal vectors and thus lie in the tangent hyperplane around point $\overline{\mathbf{b}}$, making them isovectors. We choose the isovectors that result in a zero dot product with the normal vectors by means of the least possible number of algebraic computations. The isovectors must also be linearly independent, and for this reason, we rely on the standard basis vectors to the extent possible. The computations that follow put the above discussion in mathematical form.

$$
\begin{aligned}
& \mathbf{t}^{\sigma} \cdot \nabla_{\mathbf{b}} g^{1}\left(\mathbf{x}_{M+2} ; \mathbf{b}\right)=\left(\mathbf{e}_{M+2}^{\sigma},-\frac{x_{\sigma}}{x_{M+1}+\alpha x_{M+2}}, 0_{M+4}, 0_{M+5}\right) \\
& \cdot\left(x_{1}, \ldots, x_{M}, 0_{M+1}, 0_{M+2}, x_{M+1}+\alpha x_{M+2}, s x_{M+2}, 0_{M+5}\right)=0, \\
& \mathbf{t}^{M+1} \cdot \nabla_{\mathbf{b}} g^{1}\left(\mathbf{x}_{M+2} ; \mathbf{b}\right)=\mathbf{e}_{M+5}^{M+1} \cdot\left(x_{1}, \ldots, x_{M}, 0_{M+1}, 0_{M+2}, x_{M+1}+\alpha x_{M+2}, s x_{M+2}, 0_{M+5}\right)=0, \\
& \mathbf{t}^{M+2} \cdot \nabla_{\mathbf{b}} g^{1}\left(\mathbf{x}_{M+2} ; \mathbf{b}\right)=\mathbf{e}_{M+5}^{M+2} \cdot\left(x_{1}, \ldots, x_{M}, 0_{M+1}, 0_{M+2}, x_{M+1}+\alpha x_{M+2}, s x_{M+2}, 0_{M+5}\right)=0, \\
& \mathbf{t}^{M+3} \cdot \nabla_{\mathbf{b}} g^{1}\left(\mathbf{x}_{M+2} ; \mathbf{b}\right)=\left(\mathbf{0}_{M+2},-\frac{s x_{M+2}}{x_{M+1}+\alpha x_{M+2}}, 1_{M+4}, 0_{M+5}\right) \\
& \cdot\left(x_{1}, \ldots, x_{M}, 0_{M+1}, 0_{M+2}, x_{M+1}+\alpha x_{M+2}, s x_{M+2}, 0_{M+5}\right)=0 .
\end{aligned}
$$


Note that the last component of $\mathbf{t}^{\sigma^{\prime}}$ for $\sigma^{\prime}=1,2, \ldots, M+3$ is zero, thereby ensuring that $\mathbf{t}^{\sigma^{\prime}} \cdot \nabla_{\mathbf{b}} g^{2}\left(\mathbf{x}_{M+2} ; \bar{E}\right)=0$ for $\sigma^{\prime}=1,2, \ldots, M+3$. Hence the set of vectors $\mathbf{t}^{\sigma^{\prime}}, \sigma^{\prime}=1,2, \ldots, M+3$ is indeed a set of isovectors, as the $M+3$ vectors are orthogonal to both of the gradient vectors of the constraint functions $\nabla_{\mathbf{b}} g^{k}\left(\mathbf{x}_{M+2} ; \mathbf{b}\right), k=1,2$ and thus lie in the tangent hyperplane.

The third step involves computing the GCDs by taking the dot product of the isovectors with the gradient operator. For example, the first $M$ GCDs are obtained as follows:

$$
\begin{aligned}
D_{\sigma}\left(\mathbf{x}_{M+2}, \mathbf{b}\right) \stackrel{\text { def }}{=} \mathbf{t}^{\sigma} \cdot \nabla_{\mathbf{b}}= & \left(\mathbf{e}_{M+2}^{\sigma},-\frac{x_{\sigma}}{x_{M+1}+\alpha x_{M+2}}, 0_{M+4}, 0_{M+5}\right) \\
& \cdot\left(\frac{\partial}{\partial w_{1}}, \frac{\partial}{\partial w_{2}}, \ldots, \frac{\partial}{\partial w_{M+2}}, \frac{\partial}{\partial s}, \frac{\partial}{\partial \alpha}, \frac{\partial}{\partial \bar{E}}\right)=\frac{\partial}{\partial w_{\sigma}}-\frac{x_{\sigma}}{x_{M+1}+\alpha x_{M+2}} \frac{\partial}{\partial s},
\end{aligned}
$$

$\sigma=1,2, \ldots, M$. By the same procedure, the remaining GCDs are

$$
D_{M+1}\left(\mathbf{x}_{M+2}, \mathbf{b}\right)=\frac{\partial}{\partial w_{M+1}}, D_{M+2}\left(\mathbf{x}_{M+2}, \mathbf{b}\right)=\frac{\partial}{\partial w_{M+2}}, D_{M+3}\left(\mathbf{x}_{M+2}, \mathbf{b}\right)=\frac{\partial}{\partial \alpha}-\frac{s x_{M+2}}{x_{M+1}+\alpha x_{M+2}} \frac{\partial}{\partial s} .
$$

Because $D_{\sigma^{\prime}}\left(\mathbf{x}_{M+2}, \mathbf{b}\right) \circ g^{k}\left(\mathbf{x}_{M+2} ; \mathbf{b}\right)=0$ for $\sigma^{\prime}=1,2, \ldots, M+3$ and $k=1,2$, the GCDs are said to posses the null property, a property of the GCDs that distinguishes them from other directional derivatives. As Partovi and Caputo stated (2006, pp. 38-39), "Their [the GCDs] null property simply reflects the fact that the rate of change of a function is zero in directions tangent to its level surface [in parameter space]. Moreover, the reason for making the null property a defining characteristic of GCDs is the crucial fact that when the GCDs possess this property with respect to the constraint functions, the resulting semidefiniteness comparative statics results emerge free of constraints."

In the fourth step, Theorem 1 of Partovi and Caputo $(2006,2007)$ instructs us to apply the GCDs to the first-order necessary conditions, then to substitute the obtained expressions directly into the equation prescribed by their Theorem 1. If both constraints bind, as they do by supposition (A.4), the first-order necessary conditions are given by Eqs. (5)-(9), with the first terms of Eqs. (8) and (9) holding as equalities. 
Applying the GCDs to each of the first-order necessary conditions yields the following set of results for $\sigma, l=1,2, \ldots, M$ :

$$
\begin{aligned}
& D_{\sigma}\left(\mathbf{x}_{M+2}^{*}(\mathbf{b}), \mathbf{b}\right) \circ \frac{\partial L}{\partial x_{l}}\left(\mathbf{x}_{M+2}^{*}(\mathbf{b}), \lambda_{1}^{*}(\mathbf{b}), \lambda_{2}^{*}(\mathbf{b}) ; \mathbf{b}\right)=\left(\frac{\partial}{\partial w_{\sigma}}-\frac{x_{\sigma}^{*}(\mathbf{b})}{x_{M+1}^{*}(\mathbf{b})+\alpha x_{M+2}^{*}(\mathbf{b})} \frac{\partial}{\partial s}\right) \\
& \left.\circ\left[\left[1-\lambda_{1}\right]\left[R_{x_{l}}\left(\mathbf{x}_{M+1}\right)-w_{l}\right]-\lambda_{2} h_{y}\left(f\left(\mathbf{x}_{M+1}\right), x_{M+2}\right) f_{x_{l}}\left(\mathbf{x}_{M+1}\right)\right]\right|_{\substack{\mathbf{x}_{M+2}^{*}(\mathbf{b}) \\
\lambda_{i}^{*}(\mathbf{b}), i=1,2}} \\
& =\left\{\begin{array}{cc}
-\left[1-\lambda_{1}^{*}(\mathbf{b})\right] & \text { if } \sigma=l \\
0 & \text { otherwise }
\end{array}\right. \\
& D_{\sigma}\left(\mathbf{x}_{M+2}^{*}(\mathbf{b}), \mathbf{b}\right) \circ \frac{\partial L}{\partial x_{M+1}}\left(\mathbf{x}_{M+2}^{*}(\mathbf{b}), \lambda_{1}^{*}(\mathbf{b}), \lambda_{2}^{*}(\mathbf{b}) ; \mathbf{b}\right)=\left(\frac{\partial}{\partial w_{\sigma}}-\frac{x_{\sigma}^{*}(\mathbf{b})}{x_{M+1}^{*}(\mathbf{b})+\alpha x_{M+2}^{*}(\mathbf{b})} \frac{\partial}{\partial s}\right) \\
& \left.\circ\left[\left[R_{x_{M+1}}\left(\mathbf{x}_{M+1}\right)-w_{M+1}\right]+\lambda_{1}\left[s-R_{x_{M+1}}\left(\mathbf{x}_{M+1}\right)\right]-\lambda_{2} h_{y}\left(f\left(\mathbf{x}_{M+1}\right), x_{M+2}\right) f_{x_{M+1}}\left(\mathbf{x}_{M+1}\right)\right]\right|_{\substack{\mathbf{x}_{M+2}^{*}(\mathbf{b}) \\
\lambda_{i}^{*}(\mathbf{b}), i=1,2}} \\
& =-\lambda_{1}^{*}(\mathbf{b}) \frac{x_{\sigma}^{*}(\mathbf{b})}{x_{M+1}^{*}(\mathbf{b})+\alpha x_{M+2}^{*}(\mathbf{b})}, \\
& D_{\sigma}\left(\mathbf{x}_{M+2}^{*}(\mathbf{b}), \mathbf{b}\right) \circ \frac{\partial L}{\partial x_{M+2}}\left(\mathbf{x}_{M+2}^{*}(\mathbf{b}), \lambda_{1}^{*}(\mathbf{b}), \lambda_{2}^{*}(\mathbf{b}) ; \mathbf{b}\right)=\left(\frac{\partial}{\partial w_{\sigma}}-\frac{x_{\sigma}^{*}(\mathbf{b})}{x_{M+1}^{*}(\mathbf{b})+\alpha x_{M+2}^{*}(\mathbf{b})} \frac{\partial}{\partial s}\right) \\
& \left.\circ\left[-w_{M+2}+\lambda_{1} \alpha s-\lambda_{2} h_{x_{M+2}}\left(f\left(\mathbf{x}_{M+1}\right), x_{M+2}\right)\right)\right]\left.\right|_{\substack{\mathbf{x}_{M+2}^{*}(\mathbf{b}) \\
\lambda_{i}^{*}(\mathbf{b}), i=1,2}} \\
& =-\alpha \lambda_{1}^{*}(\mathbf{b}) \frac{x_{\sigma}^{*}(\mathbf{b})}{x_{M+1}^{*}(\mathbf{b})+\alpha x_{M+2}^{*}(\mathbf{b})}, \\
& D_{M+1}\left(\mathbf{x}_{M+2}^{*}(\mathbf{b}), \mathbf{b}\right) \circ \frac{\partial L}{\partial x_{l}}\left(\mathbf{x}_{M+2}^{*}(\mathbf{b}), \lambda_{1}^{*}(\mathbf{b}), \lambda_{2}^{*}(\mathbf{b}) ; \mathbf{b}\right) \\
& =\left.\frac{\partial}{\partial w_{M+1}} \circ\left[\left[1-\lambda_{1}\right]\left[R_{x_{l}}\left(\mathbf{x}_{M+1}\right)-w_{l}\right]-\lambda_{2} h_{y}\left(f\left(\mathbf{x}_{M+1}\right), x_{M+2}\right) f_{x_{l}}\left(\mathbf{x}_{M+1}\right)\right]\right|_{\substack{\mathbf{x}_{M+2}^{*}(\mathbf{b}) \\
\lambda_{i}^{*}(\mathbf{b}), i=1,2}}=0 \\
& D_{M+1}\left(\mathbf{x}_{M+2}^{*}(\mathbf{b}), \mathbf{b}\right) \circ \frac{\partial L}{\partial x_{M+1}}\left(\mathbf{x}_{M+2}^{*}(\mathbf{b}), \lambda_{1}^{*}(\mathbf{b}), \lambda_{2}^{*}(\mathbf{b}) ; \mathbf{b}\right)=-1, \\
& D_{M+1}\left(\mathbf{x}_{M+2}^{*}(\mathbf{b}), \mathbf{b}\right) \circ \frac{\partial L}{\partial x_{M+2}}\left(\mathbf{x}_{M+2}^{*}(\mathbf{b}), \lambda_{1}^{*}(\mathbf{b}), \lambda_{2}^{*}(\mathbf{b}) ; \mathbf{b}\right)=0 \\
& D_{M+2}\left(\mathbf{x}_{M+2}^{*}(\mathbf{b}), \mathbf{b}\right) \circ \frac{\partial L}{\partial x_{l}}\left(\mathbf{x}_{M+2}^{*}(\mathbf{b}), \lambda_{1}^{*}(\mathbf{b}), \lambda_{2}^{*}(\mathbf{b}) ; \mathbf{b}\right)=0
\end{aligned}
$$




$$
\begin{aligned}
& D_{M+2}\left(\mathbf{x}_{M+2}^{*}(\mathbf{b}), \mathbf{b}\right) \circ \frac{\partial L}{\partial x_{M+1}}\left(\mathbf{x}_{M+2}^{*}(\mathbf{b}), \lambda_{1}^{*}(\mathbf{b}), \lambda_{2}^{*}(\mathbf{b}) ; \mathbf{b}\right)=0 \\
& D_{M+2}\left(\mathbf{x}_{M+2}^{*}(\mathbf{b}), \mathbf{b}\right) \circ \frac{\partial L}{\partial x_{M+2}}\left(\mathbf{x}_{M+2}^{*}(\mathbf{b}), \lambda_{1}^{*}(\mathbf{b}), \lambda_{2}^{*}(\mathbf{b}) ; \mathbf{b}\right)=-1, \\
& D_{M+3}\left(\mathbf{x}_{M+2}^{*}(\mathbf{b}), \mathbf{b}\right) \circ \frac{\partial L}{\partial x_{l}}\left(\mathbf{x}_{M+2}^{*}(\mathbf{b}), \lambda_{1}^{*}(\mathbf{b}), \lambda_{2}^{*}(\mathbf{b}) ; \mathbf{b}\right)=\left(\frac{\partial}{\partial \alpha}-\frac{s x_{M+2}^{*}(\mathbf{b})}{x_{M+1}^{*}(\mathbf{b})+\alpha x_{M+2}^{*}(\mathbf{b})} \frac{\partial}{\partial s}\right) \\
& \left.\circ\left[\left[1-\lambda_{1}\right]\left[R_{x_{l}}\left(\mathbf{x}_{M+1}\right)-w_{l}\right]-\lambda_{2} h_{y}\left(f\left(\mathbf{x}_{M+1}\right), x_{M+2}\right) f_{x_{l}}\left(\mathbf{x}_{M+1}\right)\right]\right|_{\substack{\mathbf{x}_{M+2}^{*}(\mathbf{b}) \\
\lambda_{i}^{*}(\mathbf{b}), i=1,2}}=0, \\
& D_{M+3}\left(\mathbf{x}_{M+2}^{*}(\mathbf{b}), \mathbf{b}\right) \circ \frac{\partial L}{\partial x_{M+1}}\left(\mathbf{x}_{M+2}^{*}(\mathbf{b}), \lambda_{1}^{*}(\mathbf{b}), \lambda_{2}^{*}(\mathbf{b}) ; \mathbf{b}\right)=\left(\frac{\partial}{\partial \alpha}-\frac{s x_{M+2}^{*}(\mathbf{b})}{x_{M+1}^{*}(\mathbf{b})+\alpha x_{M+2}^{*}(\mathbf{b})} \frac{\partial}{\partial s}\right) \\
& \left.\circ\left[\left[R_{x_{M+1}}\left(\mathbf{x}_{M+1}\right)-w_{M+1}\right]+\lambda_{1}\left[s-R_{x_{M+1}}\left(\mathbf{x}_{M+1}\right)\right]-\lambda_{2} h_{y}\left(f\left(\mathbf{x}_{M+1}\right), x_{M+2}\right) f_{x_{M+1}}\left(\mathbf{x}_{M+1}\right)\right]\right|_{\substack{\mathbf{x}_{M+2}^{*}(\mathbf{b}) \\
\lambda_{i}^{M}(\mathbf{b}), i=1,2}} \\
& =-\lambda_{1}^{*}(\mathbf{b}) \frac{s x_{M+2}^{*}(\mathbf{b})}{x_{M+1}^{*}(\mathbf{b})+\alpha x_{M+2}^{*}(\mathbf{b})}, \\
& D_{M+3}\left(\mathbf{x}_{M+2}^{*}(\mathbf{b}), \mathbf{b}\right) \circ \frac{\partial L}{\partial x_{M+2}}\left(\mathbf{x}_{M+2}^{*}(\mathbf{b}), \lambda_{1}^{*}(\mathbf{b}), \lambda_{2}^{*}(\mathbf{b}) ; \mathbf{b}\right)=\left(\frac{\partial}{\partial \alpha}-\frac{s x_{M+2}^{*}(\mathbf{b})}{x_{M+1}^{*}(\mathbf{b})+\alpha x_{M+2}^{*}(\mathbf{b})} \frac{\partial}{\partial s}\right) \\
& \left.\circ\left[-w_{M+2}+\lambda_{1} \alpha s-\lambda_{2} h_{x_{M+2}}\left(f\left(\mathbf{x}_{M+1}\right), x_{M+2}\right)\right]\right|_{\substack{\mathbf{x}_{M+2}^{*}(\mathbf{b}) \\
\lambda_{i}^{M+(\mathbf{b}), i=1,2}}} \\
& =\lambda_{1}^{*}(\mathbf{b}) s-\lambda_{1}^{*}(\mathbf{b}) \alpha \frac{s x_{M+2}^{*}(\mathbf{b})}{x_{M+1}^{*}(\mathbf{b})+\alpha x_{M+2}^{*}(\mathbf{b})}=\lambda_{1}^{*}(\mathbf{b}) \frac{s x_{M+1}^{*}(\mathbf{b})}{x_{M+1}^{*}(\mathbf{b})+\alpha x_{M+2}^{*}(\mathbf{b})} .
\end{aligned}
$$

According to Theorem 1 of Partovi and Caputo (2006), the typical element of $\Omega(\mathbf{b})$ is

$$
\Omega_{\xi \xi^{\prime}}(\mathbf{b})=-\sum_{n=1}^{M+2}\left[D_{\xi}\left(\mathbf{x}_{M+2}^{*}(\mathbf{b}), \mathbf{b}\right) \circ \frac{\partial L}{\partial x_{n}}\left(\mathbf{x}_{M+2}^{*}(\mathbf{b}), \lambda_{1}^{*}(\mathbf{b}), \lambda_{2}^{*}(\mathbf{b}) ; \mathbf{b}\right)\right]\left[D_{\xi^{\prime}}\left(\mathbf{x}_{M+2}^{*}(\mathbf{b}), \mathbf{b}\right) \circ x_{n}^{*}(\mathbf{b})\right],
$$

where $\xi, \xi^{\prime}=1,2, \ldots, M+3$. The negative sign in the above expression, added for closer resemblance to the work of Caputo and Partovi (2008), has the effect of changing the sign of each element of the matrix. Consequently, it changes the semidefiniteness of the matrix from positive to negative. For $\sigma, \tau \leq 1,2, \ldots, M$, the typical element of $\Omega(\mathbf{b})$ is given by 


$$
\begin{gathered}
\Omega_{\sigma, \tau}(\mathbf{b})=-\sum_{n=1}^{M+2}\left[D_{\sigma}\left(\mathbf{x}_{M+2}^{*}(\mathbf{b}), \mathbf{b}\right) \circ \frac{\partial L}{\partial x_{n}}\left(\mathbf{x}_{M+2}^{*}(\mathbf{b}), \lambda_{1}^{*}(\mathbf{b}), \lambda_{2}^{*}(\mathbf{b}) ; \mathbf{b}\right)\right]\left[D_{\tau}\left(\mathbf{x}_{M+2}^{*}(\mathbf{b}), \mathbf{b}\right) \circ x_{n}^{*}(\mathbf{b})\right] \\
=-\left[D_{\sigma}\left(\mathbf{x}_{M+2}^{*}(\mathbf{b}), \mathbf{b}\right) \circ \frac{\partial L}{\partial x_{\sigma}}\left(\mathbf{x}_{M+2}^{*}(\mathbf{b}), \lambda_{1}^{*}(\mathbf{b}), \lambda_{2}^{*}(\mathbf{b}) ; \mathbf{b}\right)\right]\left[\frac{\partial x_{\sigma}^{*}(\mathbf{b})}{\partial w_{\tau}}-\frac{x_{\tau}^{*}(\mathbf{b})}{x_{M+1}^{*}(\mathbf{b})+\alpha x_{M+2}^{*}(\mathbf{b})} \frac{\partial x_{\sigma}^{*}(\mathbf{b})}{\partial s}\right] \\
-\left[D_{\sigma}\left(\mathbf{x}_{M+2}^{*}(\mathbf{b}), \mathbf{b}\right) \circ \frac{\partial L}{\partial x_{M+1}}\left(\mathbf{x}_{M+2}^{*}(\mathbf{b}), \lambda_{1}^{*}(\mathbf{b}), \lambda_{2}^{*}(\mathbf{b}) ; \mathbf{b}\right)\right]\left[\frac{\partial x_{M+1}^{*}(\mathbf{b})}{\partial w_{\tau}}-\frac{x_{\tau}^{*}(\mathbf{b})}{x_{M+1}^{*}(\mathbf{b})+\alpha x_{M+2}^{*}(\mathbf{b})} \frac{\partial x_{M+1}^{*}(\mathbf{b})}{\partial s}\right] \\
-\left[D_{\sigma}\left(\mathbf{x}_{M+2}^{*}(\mathbf{b}), \mathbf{b}\right) \circ \frac{\partial L}{\partial x_{M+2}}\left(\mathbf{x}_{M+2}^{*}(\mathbf{b}), \lambda_{1}^{*}(\mathbf{b}), \lambda_{2}^{*}(\mathbf{b}) ; \mathbf{b}\right)\right]\left[\frac{\partial x_{M+2}^{*}(\mathbf{b})}{\partial w_{\tau}}-\frac{x_{\tau}^{*}(\mathbf{b})}{x_{M+1}^{*}(\mathbf{b})+\alpha x_{M+2}^{*}(\mathbf{b})} \frac{\partial x_{M+2}^{*}(\mathbf{b})}{\partial s}\right] \\
=\left[1-\lambda_{1}^{*}(\mathbf{b})\right]\left[\frac{\partial x_{\sigma}^{*}(\mathbf{b})}{\partial w_{\tau}}-\frac{x_{\tau}^{*}(\mathbf{b})}{x_{M+1}^{*}(\mathbf{b})+\alpha x_{M+2}^{*}(\mathbf{b})} \frac{\partial x_{\sigma}^{*}(\mathbf{b})}{\partial s}\right] \\
+\lambda_{1}^{*}(\mathbf{b}) \frac{x_{\sigma}^{*}(\mathbf{b})}{x_{M+1}^{*}(\mathbf{b})+\alpha x_{M+2}^{*}(\mathbf{b})}\left[\frac{\partial x_{M+1}^{*}(\mathbf{b})}{\partial w_{\tau}}-\frac{x_{\tau}^{*}(\mathbf{b})}{x_{M+1}^{*}(\mathbf{b})+\alpha x_{M+2}^{*}(\mathbf{b})} \frac{\partial x_{M+1}^{*}(\mathbf{b})}{\partial s}\right] \\
+\alpha \lambda_{1}^{*}(\mathbf{b}) \frac{x_{\sigma}^{*}(\mathbf{b})}{x_{M+1}^{*}(\mathbf{b})+\alpha x_{M+2}^{*}(\mathbf{b})}\left[\frac{\partial x_{M+2}^{*}(\mathbf{b})}{\partial w_{\tau}}-\frac{x_{\tau}^{*}(\mathbf{b})}{x_{M+1}^{*}(\mathbf{b})+\alpha x_{M+2}^{*}(\mathbf{b})} \frac{\partial x_{M+2}^{*}(\mathbf{b})}{\partial s}\right] .
\end{gathered}
$$

The remaining elements of the comparative statics matrix are obtained in the same way. Q.E.D. Proof of Proposition 2. For the CAC constraints listed in Proposition 2, the parameter vectors and the gradient operators differ trivially from the ones given in the proof of Theorem 1, while the set of normal vectors is identical. For example, if the monopolist faces the emissions-perunit-of-output constraint, then the parameter vector is $\mathbf{b}^{1} \stackrel{\text { def }}{=}\left(\mathbf{w}_{M+2}, s, \alpha, \bar{E}_{P F}\right) \in \mathbb{R}_{++}^{M+5}$ and the gradient operator is $\nabla_{\mathbf{b}^{1}} \stackrel{\text { def }}{=}\left(\frac{\partial}{\partial w_{1}}, \frac{\partial}{\partial w_{2}}, \ldots, \frac{\partial}{\partial w_{M+2}}, \frac{\partial}{\partial s}, \frac{\partial}{\partial \alpha}, \frac{\partial}{\partial \bar{E}_{P F}}\right)$. Define the constraint function $g^{2^{\prime}}(\cdot)$ as $g^{2^{\prime}}\left(\mathbf{x}_{M+2} ; \bar{E}_{P F}\right) \stackrel{\text { def }}{=} \bar{E}_{P F}-h\left(y, x_{M+2}\right) / y$. Then the vector normal to $g^{2^{\prime}}(\cdot)$ is given by $\nabla_{\mathbf{b}^{\prime}} g^{2^{\prime}}\left(\mathbf{x}_{M+2} ; \bar{E}_{P F}\right)=\left(\mathbf{0}_{M+4}, 1_{M+5}\right)$, which is identical to the vector normal to the fixed-level-ofemissions constraint. What's more, just like parameter $\bar{E}$, parameter $\bar{E}_{P F}$ does not enter the ROR constraint, hence the vector normal to the ROR constraint is identical for both parameters. By the same logic, the normal vectors are given by Eqs. (35) and (36) for all the CAC constraints given in Proposition 2. Since these normal vectors are the same as the normal vectors in the proof of Theorem 1, Steps 2-4 in the proof of Theorem 1 remain unchanged, and accordingly, so does the form of the comparative statics matrix.

Q.E.D. 


\section{References}

Armstrong, M. and Sappington, D.E.M. (2007). Recent developments in the theory of regulation, in Handbook of Industrial Organization (Vol. III), edited by M. Armstrong and R. Porter, North-Holland, Amsterdam, The Netherlands

Averch, H. and Johnson, L.L. (1962). Behavior of the firm under regulatory constraint, American Economic Review 52: 1052-1069.

Baron, D.P. (1985). Noncooperative regulation of a nonlocalized externality, The RAND Journal of Economics 16: 553-568.

Caputo, M.R. and Partovi, M.H. (2002). Reexamination of the A-J effect, Economics Bulletin 12: $1-9$.

Caputo, M.R. and Partovi, M.H. (2008). Intrinsic comparative statics of a general class of profitmaximizing rate-of-return regulated firms, Oxford Economic Papers 60: 369-382.

Helfand, G.E. (1991). Standards versus standards: The effects of different pollution restrictions, American Economic Review 81: 622-634.

Helfand, G.E. and Berck, P. (2003). The theory and practice of command and control in environmental policy, Ashgate Publishing Company, Burlington, VT.

Joskow, P.L. (1972). The determination of the allowed rate of return in a formal regulatory hearing, The Bell Journal of Economics and Management Science 3: 632-644.

Meran, G. and Schwalbe U. (2006). Price and quality regulation in the water industry: the need for coordination, paper presented at the 2006 parallel meetings of the European Economic Association and Econometric Society, Vienna Austria.

Partovi, M.H. and Caputo, M.R. (2006). A complete theory of comparative statics for differentiable optimization problems, Metroeconomica 57: 31-67.

Partovi, M.H. and Caputo, M.R. (2007). Erratum: a complete theory of comparative statics for differentiable optimization problems, Metroeconomica 58: 360. 
Perino, G. (2010). Price discrimination based on downstream regulation: evidence from the market for scrubbers, ccp10-09 working paper, School of Economics, University of East Anglia.

Rose, K., Burns, R.E., Coggins, J.S., Harunuzzaman, M. and Viezer, T.W. (1992). Public utility commission implementation of the Clean Air Act's allowance trading program, NRRI Publications. All Electricity Documents, pp 118-144, 245-256.

Takayama, A. (1993). Analytical methods in economics, The University of Michigan Press, Ann Arbor, MI. 